\title{
Regeneration capacity of potato cultivars prone to cold sweetening
}

\author{
Ibragimova S. ${ }^{1 *}$, Romanova A. ${ }^{1}$, Saboiev I. ${ }^{1}$, Salina E. ${ }^{1}$, Kochetov A. ${ }^{1,2}$ \\ ${ }^{1}$ Kurchatov Genomic Center of the Institute of Cytology and Genetics, SB RAS, Novosibirsk, Russia \\ ${ }^{2}$ Novosibirsk State University, Novosibirsk, Russia \\ *email: isola@bionet.nsc.ru
}

The regeneration of plant tissue culture technique is an essential component of biotechnological research, and required for the genetic manipulation of the plants. Only certain cultivars of tetraploid potato are amenable to transformation and others need to be test for transformation and regeneration in tissue culture. A successful and reproducible plant system requires a responsive in vitro regeneration system. Three potatoes cultivars Nikulinsky, Symphony and Nevsky (Solanum tuberosum L.) prone to cold sweetening were tests for their ability to regenerate in vitro. Stem explants was collect from in vitro grown plants. During the cultivation up to obtaining shoots, stem explants were cultivate on P1medium: MS medium, supplemented with $1 \mathrm{mg} / \mathrm{L}$ transzeatin, $0.1 \mathrm{mg} / \mathrm{L}$ IAA, $10 \mathrm{mg} / \mathrm{L} \mathrm{GA} 3$ and vitamins. Every two weeks the explants were transfer to fresh nutrient media. The time requirements for callus induction shoot regeneration and rooting was significantly induce by cultivars. Early callus initiation occurred in cv. Symphony after 6-x days cultivation, in cv. Nikulinsky and Nevsky after 35-45 days. Callus were differentiated into shoot-primordia when cultured on MS medium supplemented with the same hormones. Cultivar Symphony also showed early shoot initiation (after 15-20 days) and the mean number of shoots was higher than in other cultivars. Callus derived shoots were rooted most effectively in full-strength MS medium without hormone addition. Regenerated plants were morphologically uniform with normal leaf shape and growth pattern in all three cultivars. The full cycle of regeneration from the moment of callus to the receipt of regenerated plants of the cv. Symphony was 4 months, and cv. Nikulinsky and Nevsky - 5.5-6.0 months. Based on the data obtained, the cv. Symphony can be consider as a promising cultivar for the genetic manipulation.

Acknowledgments: The study is supported by the Kurchatov Genomic Center of the Institute of Cytology and Genetics, SB RAS (075-15-2019-1662). 